\title{
Purified vitexin compound 1 induces apoptosis through activation of FOXO3a in hepatocellular carcinoma
}

\author{
JIAN-GANG WANG ${ }^{1}$, XING-XING ZHENG ${ }^{1}$, GUANG-YAO ZENG $^{2}$, YING-JUN ZHOU ${ }^{2}$ and HONG YUAN HA $^{1,3}$ \\ ${ }^{1}$ Department of Cardiology, The Third Xiangya Hospital of Central South University; ${ }^{2}$ School of Pharmaceutical Science, \\ Central South University; ${ }^{3}$ Center of Clinical Pharmacology, The Third Xiangya Hospital, \\ Central South University, Changsha, Hunan 410013, P.R. China
}

Received September 13, 2013; Accepted October 29, 2013

DOI: $10.3892 /$ or.2013.2855

\begin{abstract}
We previously reported that purified vitexin compound 1 (VB1, a neolignan from the seed of Chinese herb Vitex negundo) exhibited antitumor activity in cancer cell lines and xenograft models. In the present study, we examined the molecular mechanisms by which activation of the FOXO3a transcription factor mediated VB1-induced apoptosis in hepatocellular carcinoma (HCC) cells. The effects of VB1 on the proliferation of HCC cell lines HepG2, Hep3B, Huh-7 and human embryo liver L-02 cells were investigated using the 3-(4,5-dimethylthiazol-2-yl)-2,5-diphenyltetrazolium bromide (MTT) assay. Apoptotic death in HepG2 cells was examined using an enzyme-linked immunosorbent assay (ELISA) detection kit, flow cytometry after propidium iodide (PI) staining, and by DNA agarose gel electrophoresis. Caspase activity was measured using ELISA. The AKT/FOXO3a and ERK/ FOXO3a pathways were analyzed using western blotting. VB1 inhibited human HCC cell proliferation in a concentrationdependent manner and increased the percentage of sub-G1 population HepG 2 cells. Histone/DNA fragmentation and active caspase-3, -8 and -9 levels increased in a concentrationdependent manner and a DNA ladder was formed. The phosphorylation of AKT and ERK1/2 were inhibited and FOXO3a transcription factor was activated, resulting in apoptotic death. Knockdown of AKT1 by small interfering RNA (siRNA) and the MEK1/2 inhibitor, PD98059, enhanced VB1-induced apoptosis and FOXO3a transcriptional activity. Suppression of FOXO3a expression by siRNA inhibited VB1-induced apoptosis. VB1 induced expression of Bim, TRAIL, DR4 and DR5. Activation of the FOXO3a transcription factor appears to mediate pro-apoptotic effects of VB1 by inhibiting the AKT and ERK pathways.
\end{abstract}

Correspondence to: Dr Hong Yuan, Department of Cardiology, The Third Xiangya Hospital of Central South University, Changsha, Hunan 410013, P.R. China

E-mail: yuanhong01@vip.sina.com

Key words: hepatocellular carcinoma, purified vitexin compound 1, apoptosis, AKT, ERK, FOXO3a

\section{Introduction}

Liver cancer is the fifth most common cancer worldwide and the third most common cause of cancer mortality $(1,2)$. Hepatocellular carcinoma (HCC), which accounts for $80-90 \%$ of primary liver tumors, is characterized by a poor prognosis and high mortality $(1,2)$. Despite therapeutic advances, the overall survival of patients with HCC has not significantly improved in the last two decades. Surgical resection, radiofrequency ablation (RFA) and percutaneous ethanol injection (PEI) are accompanied by high recurrence rates (3-5). Transarterial chemoembolization (TACE), the most commonly used non-surgical alternative, is usually reserved for palliative treatment (6), and chemotherapy and radiotherapy generally offer unsatisfactory response rates. Thus, new therapeutic strategies are urgently required for the treatment of HCC.

Vitexins are a group of complex polyphenolic antioxidants found in plants. Vitexin inhibits $\alpha$-glucosidase, restrains rotavirus infection and has significant protective effects against $\mathrm{CCl}_{4}$-induced hepatotoxicity (7-9). Several vitexins have been shown to induce apoptosis of human leukemia and colon cancer cells $(10,11)$. In previous studies, we demonstrated that a mixture of vitexin lignans, known as EVn-50, exhibits obvious in vitro and in vivo anticancer activity against hormone-dependent cancer and non-hormone dependent cancer such as breast, prostate, ovarian, gastric cancer and choriocarcinoma via a possible mechanism of promoting apoptosis of cancer cells (12). The purified vitexin compound 1 (VB1, a neolignan compound), which is the most abundant Vitexin compound in EVn-50, has potent cytotoxic effects in several cancer cell lines, including human HCC cells $(13,14)$. The broad-ranging antitumor effects and cytotoxicity of VB1 may be mediated by alternation of Bax/Bcl-2 ratio in favor of Bax, by activation of caspases, and by suppression of the mTOR pathway $(13,14)$. These studies strongly suggest that VB1 can be developed as a cancer preventive agent. However, to date, no studies address the effects of VB1 on the induction of apoptosis in HCC cells.

The PI3K/AKT and MEK/ERK pathways have profound effects on proliferative, apoptotic and differentiation pathways. Dysregulation of components of these cascades can contribute to resistance to other pathway inhibitors, chemotherapeutic 
drug resistance as well as other diseases (15). Overexpression of AKT and ERK have been reported in several types of human cancer, including HCC, and cells expressing elevated levels of AKT and ERK are less sensitive to apoptosis stimuli (16-18). Other well known risk factors for HCC such as HBV and HCV infection also seem to utilize the AKT and ERK pathways for the control of hepatocyte survival and viral replication (19). Furthermore, activation of AKT and ERK signaling pathways predicts poor prognosis in HCC (17), supporting the participation of activated PI3K/AKT and MEK/ERK axes in this disease.

FOXO3a is a member of the forkhead/winged helix box class $\mathrm{O}(\mathrm{FOXO})$ transcription factors that participates in a variety of cellular processes such as cell cycle progression, stress, detoxification, DNA damage repair, glucose metabolism and differentiation (20). During tumor development, inhibition of FOXO3a-induced transcriptional activity promotes cell transformation, tumor progression and angiogenesis (21-23). A number of kinases have been shown to suppress FOXO activity. These include phosphoinositide AKT (24) and extracellular signal-regulated kinases 1 and 2 (ERK1/2) (25). However, whether VB1 induces apoptosis in $\mathrm{HCC}$ cells by regulating FOXO3a transcription factor through inhibition of AKT and ERK1/2 kinases has yet to be confirmed.

In the present study, we demonstrated that the selective cytotoxicity of VB1 in HCC cells was accompanied by inhibition of AKT and ERK1/2 kinase phosphorylation, activation of FOXO3a transcription factor and apoptotic death in HepG2 cells. Furthermore, knockdown of AKT by siRNA or PD98059 enhanced VB1-induced FOXO3a transcriptional activity. These data suggest that VB1 induces apoptosis by activating FOXO3a transcription factor and that this is most likely induced by inhibition of AKT and ERK1/2 phosphorylation.

\section{Materials and methods}

Reagents. VB1 [6-hydroxy-4-(4-hydroxy-3-methoxyphenyl)-3hydro-methyl-7-methoxy-3,4-dihydro-2-naphthaldehyde] was purified from EVn-50 (a mixture of lignan compounds obtained from Vitex negundo seed) as previously described (13). 3-(4, 5-dimethylthiazol-2-yl)-2,5-diphenyltetrazolium bromide (MTT) was purchased from Sigma Chemical Co. (St. Louis, MO, USA). Fluorouracil (5-FU) was purchased from Sigma Chemical Co. The cell apoptosis enzyme-linked immunosorbent assay (ELISA) detection kit was obtained from Roche Applied Science, Mannheim, Germany. The Apoptotic DNA Ladder Detection kit was obtained from the Bodataike Company, Beijing. The Caspase-3 Activity Detection kit, Caspase-8 Colorimetric Activity Assay kit 25, and Caspase-9 Colorimetric Activity Assay kit were purchased from Millipore, Billerica, MA, USA. Caspase inhibitors, such as zVADfmk, zDEVD-fmk, zIETD-fmk and zLEHD-fmk were purchased from R\&D Systems (Minneapolis, MN, USA). Propidium iodide (PI) was purchased from Sigma Chemical Co. Antibodies against phospho-AKT, AKT, phospho-ERK1/2, ERK1/2, Bim, TRAIL, DR5, DR4, phosphor-FOXO3a, FOXO3a and $\beta$-actin were purchased from Cell Signaling Technology, Inc. (Danvers, MA, USA). Enhanced chemiluminescence (ECL) western blotting detection reagents were provided by Amersham Life Sciences Inc. (Arlington Heights, IL, USA).

Cell lines and cell culture techniques. Human HCC cell lines Hep3B, Huh-7 and HepG2 and human embryo liver L-02 cells were purchased from the China Centre for Type Culture Collection (CCTCC, Wuhan, China). The cells were maintained in Dulbecco's modified Eagle's medium (DMEM; Life Technologies, Grand Island, NY, USA) supplemented with $10 \%$ fetal bovine serum (FBS) (Invitrogen), $100 \mathrm{U} / \mathrm{ml}$ penicillin and $100 \mathrm{U} / \mathrm{ml}$ streptomycin, and cultured in a humidified atmosphere at $37^{\circ} \mathrm{C}$ with $5 \% \mathrm{CO}_{2}$.

MTT assay. Cells were seeded in a 96-well plate at a density of $0.5 \times 10^{4}$ cells/well and maintained in serum-free medium for $24 \mathrm{~h}$. This was followed by exposure to various concentrations of experimental agents that were added to each well and cultured for $24 \mathrm{~h}$, prior to incubation with media containing $5 \mathrm{mg} / \mathrm{ml} \mathrm{MTT}$ for $4 \mathrm{~h}$. The cell suspension was then centrifuged and $100 \mu \mathrm{l}$ DMSO was added to the resulting supernatant. Absorbance at the $570 \mathrm{~nm}$ wavelength $\left(\mathrm{A}_{570}\right)$ was measured using an enzyme-labeling instrument (ELX-800 type; BioTek, Shanghai, China). Relative cell viability inhibition rate was calculated as: (average $\mathrm{A}_{570}$ of the experimental group/average $\mathrm{A}_{570}$ of the control group) $\mathrm{x} 100 \%$.

DNA analysis. DNA content was analyzed by flow cytometry. Cancer cells with or without prior exposure to VB1 were collected by centrifugation and adjusted to $3 \times 10^{6}$ cells $/ \mathrm{ml}$. Pre-chilled absolute methanol was added to $0.5 \mathrm{ml}$ of cell suspension and incubated overnight at $4^{\circ} \mathrm{C}$. The methanol was removed by centrifugation. DNA was stained with PI staining solution (100 $\mu \mathrm{g} / \mathrm{ml}$ PI, $0.1 \%$ Triton $\mathrm{X}, 1 \mathrm{mM}$ EDTA in PBS) in the presence of an equal volume of DNase-free RNase $(200 \mu \mathrm{g} / \mathrm{ml})$. DNA was analyzed immediately using a Partec CyFlow ML cytometer (FACS 420; Becton-Dickinson, USA). Debris and clumps were excluded by monitoring DNA area (FL3-A) and peaks (i.e. signal height, FL3-W). Fluorescence signals from 10,000 gated cells were collected.

Histone/DNA ELISA detection of apoptosis. An ELISA kit was used to detect apoptosis in cells treated with VB1 according to the manufacturer's protocol. Briefly, cells were seeded in a 96-well plate at a density of $1 \times 10^{4}$ cells/well for $24 \mathrm{~h}$. The testing agents were then added to culture medium containing $10 \%$ FBS. After $24 \mathrm{~h}$, the cytoplasm of the control and experimental groups was transferred to a 96-well plate pre-coated with streptavidin that had been previously incubated with the biotinylated histone antibody and peroxidase-tagged mouse anti-human DNA for $2 \mathrm{~h}$ at room temperature. Absorbance was measured at $405 \mathrm{~nm}$, using an EXL-800-type enzymelinked immunosorbent apparatus (BioTek).

DNA agarose gel electrophoresis. The cells maintained in serum-free medium for $24 \mathrm{~h}$ were exposed to media containing various concentrations of experimental agents for $48 \mathrm{~h}$. The cells were washed twice with PBS, and DNA was extracted using an apoptotic DNA ladder detection kit according to the manufacturer's instructions. The extracted DNA was maintained at $4^{\circ} \mathrm{C}$ overnight. The DNA $(8.5 \mu \mathrm{l})$ was then mixed with 
$1.5 \mu \mathrm{l}$ of $6 \mathrm{X}$ buffer solution and electrophoresed on a $20 \mathrm{~g} / \mathrm{l}$ agarose gel containing ethidium bromide at $40 \mathrm{~V}$. The results were observed using a DBT-08 gel image analysis system.

Analysis of caspase-3, -8 and -9 activities. The activity of caspase $-3,-8$ and -9 was evaluated using a Caspase-3 Activity Detection kit, Caspase-8 Colorimetric Activity Assay kit 25 and Caspase-9 Colorimetric Activity Assay kit, respectively. Briefly, cell lysates were prepared after exposure to experimental agents. The assays were performed in 96-well plates by incubating $20 \mu \mathrm{g}$ of cell lysates in $100 \mu \mathrm{l}$ of reaction buffer [1\% NP-40, $20 \mathrm{mM}$ Tris- $\mathrm{HCl}(\mathrm{pH} 7.5), 137 \mathrm{mM} \mathrm{NaCl}$, $10 \%$ glycerol] containing $5 \mu \mathrm{M}$ of the caspase- 3 substrate Ac-DEVD- $p$ NA, the caspase- 8 substrate Ac-IETD- $p$ NA or the caspase-9 substrate Ac-LEHD- $p$ NA. Lysates were incubated at $37^{\circ} \mathrm{C}$ for $2 \mathrm{~h}$. Thereafter, absorbance at $405 \mathrm{~nm}$ was measured with an enzyme-labeling instrument (ELX-800 type). In the caspase inhibitor assays, cells were exposed to caspase inhibitors (i.e. $20 \mu \mathrm{M}$ zVAD-fmk, zDEVD-fmk, zIETD-fmk or $\mathrm{zLEHD}$-fmk) for $1 \mathrm{~h}$ prior to the addition of the agents tested.

Western blot analysis. Western blotting was performed as previously described (1). In brief, cells were lysed in RIPA buffer containing $1 \mathrm{X}$ protease inhibitor cocktail, and protein concentrations were determined using the Bradford assay (Bio-Rad, Philadelphia, PA, USA). Proteins were separated using 10-12.5\% SDS-PAGE and transferred to membranes (Millipore, Bedford, MA, USA) in a Tris $(20 \mathrm{mM})$, glycine $(150 \mathrm{mM})$ and methanol $(20 \%)$ buffer at $55 \mathrm{~V}$ for $4 \mathrm{~h}$ at $4^{\circ} \mathrm{C}$. After blocking in 5\% nonfat dry milk in TBS, the membranes were incubated with primary antibodies at 1:1,000 dilution in TBS overnight at $4^{\circ} \mathrm{C}$. They were then washed 3 times with TBS Tween-20, and incubated with secondary antibodies (conjugated with horseradish peroxidase at 1:5,000 dilution in TBS) for $1 \mathrm{~h}$ at room temperature. The membranes were washed again 3 times in TBS Tween-20 at room temperature. Protein bands were visualized on X-ray film using an enhanced chemiluminescence detection system.

DNA transfection. The small interfering RNA (siRNA) duplexes targeting the sequence 5'-UAAUGUGCCCGUCCU UGUCUU-3' of the human AKT1 gene, the sequence 5'-ACUCCGGGUCCAGCUCCAC-3' of the FOXO3A gene and control siRNA oligonucleotides were purchased from Dharmacon Research, Inc. (Lafayette, CO, USA). To silence Akt or FOXO3a, HepG2 cells were transfected with double stranded siRNA of Akt or FOXO3a using Signal Silence siRNA kit from Cell Signaling Technology, Inc. (Beverly, MA, USA). Briefly, $10^{6}$ cancer cells were plated in a $60-\mathrm{mm}$ petri dish for $24 \mathrm{~h} \mathrm{in} 3 \mathrm{ml}$ of transfection medium containing $20 \mu \mathrm{g}$ Lipofectamine and $100 \mathrm{nM}$ siRNA for $24 \mathrm{~h}$. Gene silencing in transfected cells was confirmed by western blotting.

Statistical analysis. Statistical analysis was performed using PRISM statistical analysis software (GrafPad Software, Inc., San Diego, CA, USA). Data are presented as means and standard deviations $( \pm \mathrm{SD})$. Differences between groups were analyzed by one- or two-way analysis of variance (ANOVA), followed by Bonferoni's multiple comparison tests. Values of $\mathrm{P}<0.05$ were considered to indicate statistically significant differences.

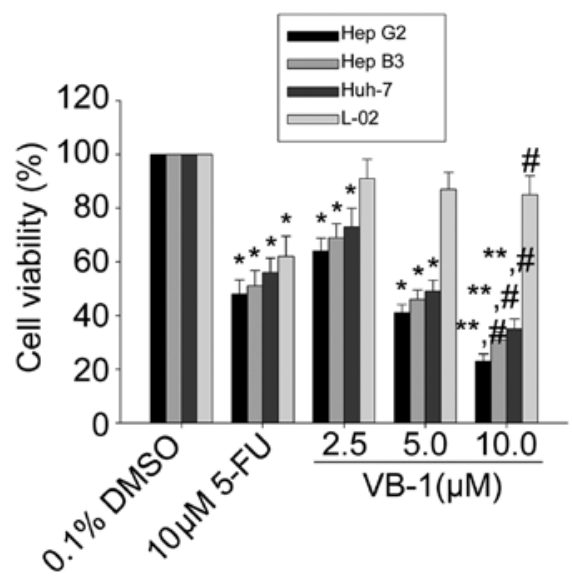

Figure 1. VB1 inhibits HCC cell viability. HepG2, Hep3B, Huh-7 and human embryo liver L-02 cells were exposed to VB1 $(0-10 \mu \mathrm{M})$ for $24 \mathrm{~h}$. Cell viability was measured by MTT assay. Data shown as means $\pm \mathrm{SD}, \mathrm{n}=3 .{ }^{*} \mathrm{P}<0.05$ when compared with respective controls; ${ }^{* *} \mathrm{P}<0.01$ when compared with respective controls; ${ }^{\#} \mathrm{P}<0.05$ when compared with the $10 \mu \mathrm{M}$ fluorouracil (5-FU) group. VB1, purified vitexin compound 1; HCC, hepatocellular carcinoma.

\section{Results}

Effects of VBI on the proliferation of human HCC cells. Since VB1 significantly inhibited the growth of several cancer cell lines, including HCC cells (13), we first examined the effects of VB1 on cell viability in 3 HCC cell lines HepG2, Hep3B and Huh-7, using the MTT assay. Fig. 1 shows that VB1 inhibited cell viability in a concentration-dependent manner. The HepG2 cell line was the most sensitive, the Hep3B cell line was moderately sensitive and the Huh-7 cell line was the least sensitive. VB1 had little effect on the human embryo liver L-02 cell line. These data suggest that VB1 is able to inhibit HCC cell growth.

Effects of VB1 on apoptosis of HepG2 cells. VB1 has been reported to induce apoptosis in breast cancer cell lines (13). We, therefore, investigated apoptosis using flow cytometric analysis to detect increases in hypodiploid cell populations. Fig. 2A shows that VB1 increased the percentage of the sub-G1 population of HepG2 cells in a concentration-dependent manner $(\mathrm{P}<0.05)$. We also showed that the histone/DNA fragment of HepG2 cells increased $(\mathrm{P}<0.05)$ in a dosedependent manner following exposure to VB1 (Fig. 2B). DNA fragmentation analysis by agarose gel electrophoresis showed a typical ladder pattern of inter-nucleosomal DNA fragments in HepG2 cells exposed to VB1 (5 or $10 \mu \mathrm{mol} / \mathrm{l}$ ) for $24 \mathrm{~h}$ (Fig. 2C). These results suggest that VB1 inhibits HCC cell growth, in part through a mechanism involving the induction of apoptosis.

Effects of VB1 on caspase activities of HepG2 cells. Most chemopreventive agents induce apoptosis through the mitochondrial pathway. To determine the effectors involved in VB1-induced apoptotic pathways, we examined whether caspases were activated during VB1-induced apoptotic death of HepG2 cells. Fig. 3A,C and E show that exposure of HepG2 cells to VB1 increased the levels of active caspase-3, -8 and -9 in a concentration-dependent manner $(\mathrm{P}<0.05)$. 
A

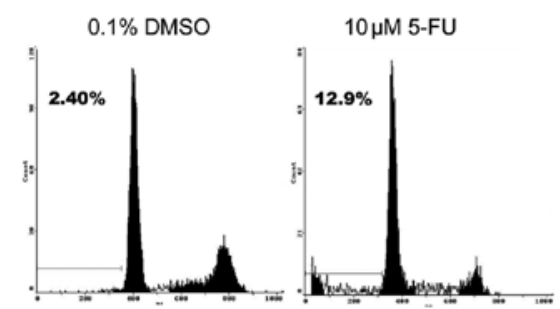

VB-1 $(\mu \mathrm{M})$

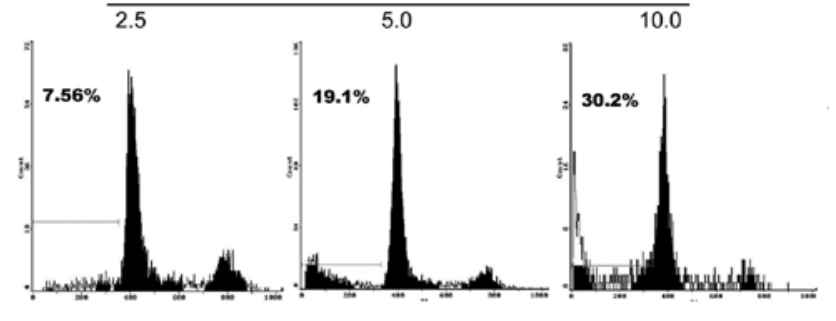

B

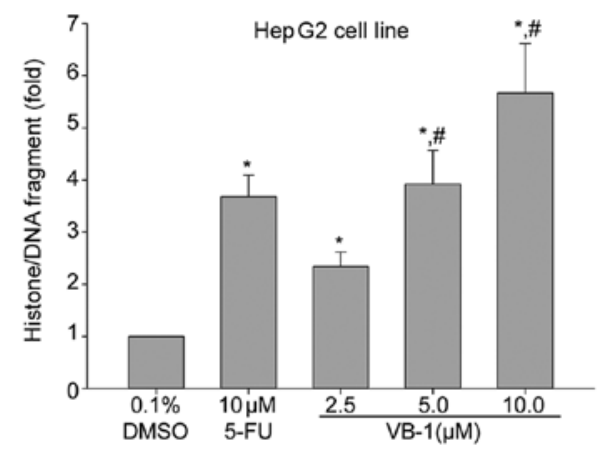

C

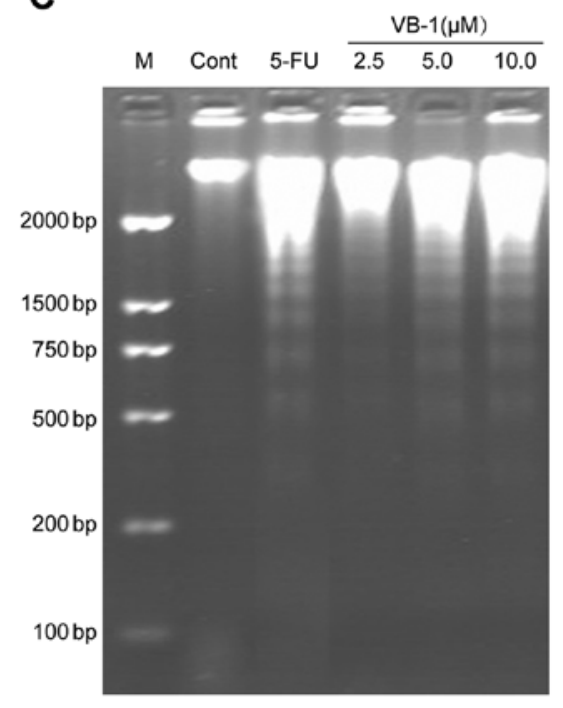

Figure 2. VB1 induces HepG2 cell apoptosis. (A) Cells were exposed to VB1 $(0-10 \mu \mathrm{M})$ for $24 \mathrm{~h}$. The apoptotic rate (the population of cells in sub-G1 phase) was examined by FCM analysis. (B) Histone/DNA fragment measured by the cell apoptosis ELISA detection kit. (C) DNA agarose gel electrophoresis. Data shown as means $\pm \mathrm{SD}, \mathrm{n}=3 .{ }^{*} \mathrm{P}<0.05$ when compared with respective controls; ${ }^{\text {P }}<0.05$ when compared with the $10 \mu \mathrm{M}$ fluorouracil (5-FU) group. M, DNA marker (DL2000). VB1, purified vitexin compound 1; FCM, flow cytometry.
We next examined the effects of pan-caspase inhibitor zVAD-fmk, the caspase-3 inhibitor zDEVD-fmk, the caspase-8 inhibitor zIETD-fmk and the caspase-9 inhibitor zLEHD-fmk on VB1-induced apoptosis. The results in Fig. 3B,D and F show that zVAD-fmk abrogated apoptosis induced by VB1, and that zDEVD-fmk zIETD-fmk and zLEHD-fmk attenuated VB1-induced apoptosis. These data indicate that VB1-induced apoptosis was dependent on the activation of caspase-3,-8 and -9 .

Effects of VBI on the phosphorylated levels of AKT and ERK1/2 proteins in the HCC cell line HepG2. AKT is constitutively active in most cancer cells and promotes cell survival and apoptosis resistance (24). We, therefore, examined the effects of VB1 on AKT phosphorylation. HepG2 cells were exposed to VB1, and phosphorylation of AKT was examined by western blot analysis. VB1 significantly inhibited AKT phosphorylation in HepG2 cells, but had no effect on total expression of AKT (Fig. 4A). These data suggest that VB1 can inhibit the phosphorylation of PI3K/AKT proteins, which may play a major role in mediating its anti-apoptotic effects.

ERK1/2 kinase regulates cellular activities ranging from gene expression to mitosis, movement, metabolism and apoptosis (25). Hence, we examined the effects of VB1 on the activation of ERK1/2 kinases. Fig. 4B shows that exposure of HepG2 cells to VB1 resulted in a decrease in ERK phosphorylated protein. These results suggest that VB1 may be involved in the regulation of ERK1/2 kinase activity.

Inhibition of AKT and ERK1/2 kinases enhances VB1-induced apoptosis. We used a siRNA that specifically silences AKT to investigate the ability of AKT to induce apoptosis by VB1. Expression of siRNAs has previously been shown to silence gene expression resulting in functional inactivation of the targeted gene $(26,27)$. Western blot analysis showed that AKT was downregulated after transfection with specific siRNA targeting AKT in HepG2 cells (Fig. 5A). The results in Fig. 5B show that inhibition of AKT by siRNA increased VB1-induced apoptosis in HepG2 cells. These results suggest that VB1-induced AKT inhibition may contribute to apoptosis of HepG2 cells.

We next examined whether VB1 induces apoptosis through inhibition of ERK1/2. HepG2 cells were exposed to $5 \mu \mathrm{M}$ VB1 for $24 \mathrm{~h}$ in the presence or absence of $20 \mu \mathrm{M}$ PD98059. The MEK1/2 inhibitor (PD98059) inhibited ERK1/2 phosphorylation and enhanced VB1-induced apoptosis in HepG2 cells (Fig. 5C and D). These data suggest that VB1-induced apoptosis may be mediated by the MEK/ERK pathway, and that inhibition of the MEK/ERK pathway enhances VB1-induced apoptosis.

Inhibition of AKT and ERK1/2 kinases enhances VB1-induced FOXO3a transcriptional activity. We next examined whether inhibition of AKT and ERK1/2 kinases regulates FOXO3a activity in the presence or absence of VB1. Knockdown of AKT by siRNA and MEK1/2 inhibitor (PD98059) inhibited the phosphorylation of FOXO3a in the presence or absence of VB1. Furthermore, knockdown of AKT by siRNA or PD98059 enhanced VB1-induced FOXO3a transcriptional activity (Fig. 6A and B). These data suggest that inhibition of AKT or ERK1/2 kinases acts synergistically with VB1 to induce FOXO3a transcriptional activity in the HCC cell line HepG2. 
A

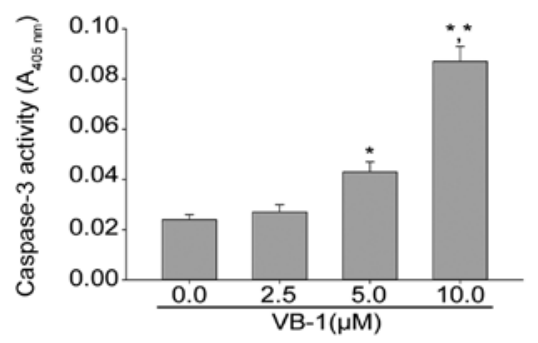

C

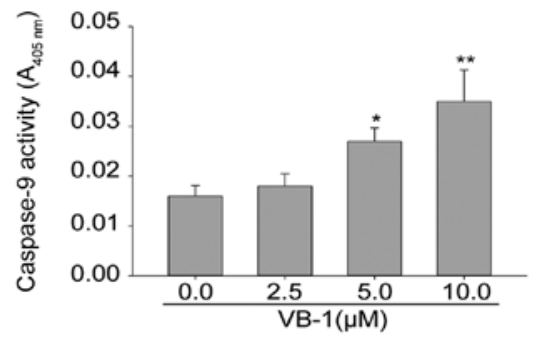

$\mathbf{E}$

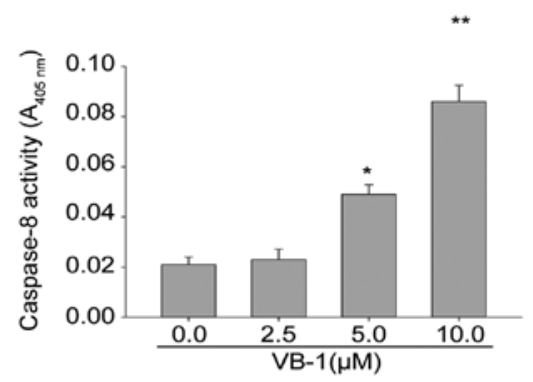

B
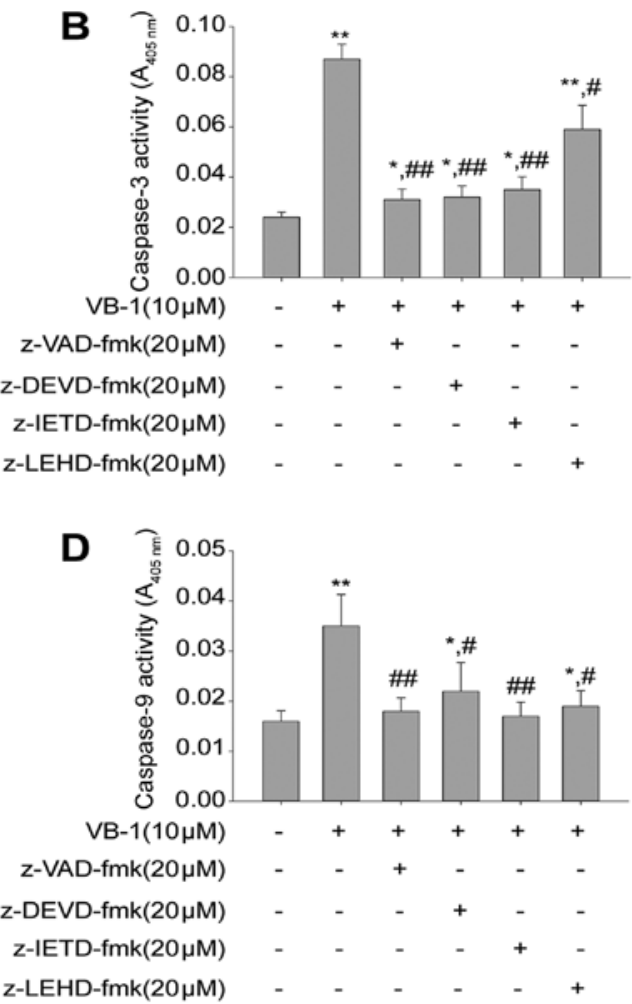

$\mathbf{F}$

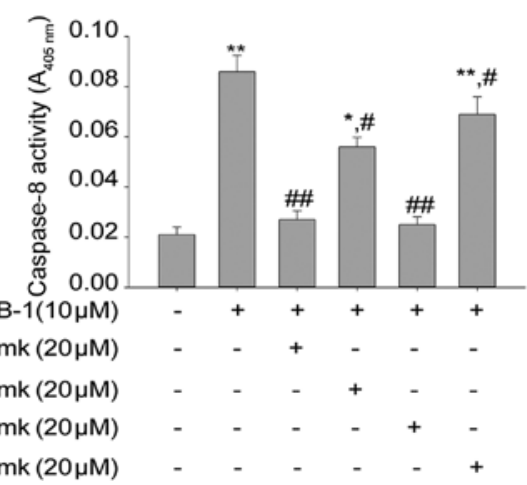

Figure 3. VB1 activates HepG2 cell caspase-3, -8 and -9. (A and B) HepG2 cells were exposed to VB1 (0-10 $\mu \mathrm{M})$ for $24 \mathrm{~h}$. The enzymatic activities of caspase-3 were determined by incubation of $20 \mu \mathrm{g}$ of total protein with $200 \mu \mathrm{mol} / 1$ chromogenic substrate (Ac-DEVD- $p \mathrm{NA}$ ) in $100 \mu \mathrm{l}$ of assay buffer for $2 \mathrm{~h}$ at $37^{\circ} \mathrm{C}$. The release of chromophore $p$-nitroanilide $(p \mathrm{NA})$ was monitored by a spectrophotometer $(405 \mathrm{~nm})$. Data shown as means $\pm \mathrm{SD}(\mathrm{n}=3)$. $\mathrm{P}<0.05$, ${ }^{* *} \mathrm{P}<0.01$ vs. $0.1 \%$ DMSO; ${ }^{\mathrm{P}}<0.05,{ }^{\# \#} \mathrm{P}<0.01$ vs. treatment with $10.0 \mu \mathrm{M}$ VB1. (C and D) HepG2 cells were exposed to VB1 (0-10 $\left.\mu \mathrm{M}\right)$ for $24 \mathrm{~h}$. The enzymatic activities of caspase- 9 were determined by incubation of $20 \mu \mathrm{g}$ of total protein with $200 \mu \mathrm{mol} / 1$ chromogenic substrate (Ac-LEHD- $p$ NA) in $100 \mu 1$ assay buffer for $2 \mathrm{~h}$ at $37^{\circ} \mathrm{C}$. The release of $p$ NA was monitored by a spectrophotometer $(405 \mathrm{~nm})$. Data shown as means $\pm \mathrm{SD}(\mathrm{n}=3)$. ${ }^{*} \mathrm{P}<0.05,{ }^{* * *} \mathrm{P}<0.01 \mathrm{vs} .0 .1 \% \mathrm{DMSO} ;{ }^{\#} \mathrm{P}<0.05$, ${ }^{\# \#} \mathrm{P}<0.01$ vs. $10.0 \mu \mathrm{M}$ VB1. (E and F) HepG2 cells were exposed to VB1 $(0-10 \mu \mathrm{M})$ for $24 \mathrm{~h}$. The enzymatic activities of caspase- 8 were determined by incubation of $20 \mu \mathrm{g}$ of total protein with $200 \mu \mathrm{mol} / 1$ chromogenic substrate (Ac-IETD-pNA) in $100 \mu \mathrm{l}$ assay buffer for $2 \mathrm{~h}$ at $37^{\circ} \mathrm{C}$. The release of $p \mathrm{NA}$ was monitored by a spectrophotometer $(405 \mathrm{~nm})$. Data shown as means $\pm \mathrm{SD}(\mathrm{n}=3) .{ }^{*} \mathrm{P}<0.05,{ }^{* *} \mathrm{P}<0.01 \mathrm{vs} .0 .1 \% \mathrm{DMSO} ;{ }^{\#} \mathrm{P}<0.05,{ }^{\# \#} \mathrm{P}<0.01 \mathrm{vs} .10 .0 \mu \mathrm{M} \mathrm{VB} 1$. VB1, purified vitexin compound 1 .

We next sought to examine the effects of FOXO3a transcription factor on the regulation of AKT and ERK1/2 kinases. We used a specific siRNA targeting FOXO3a in HepG2 cells. Downregulation of FOXO3a by siRNA has no effect on the expression of p-AKT, AKT, p-ERK1/2 and ERK1/2. Effects of VB1 on the phosphorylated levels of AKT and ERK1/2 proteins in the HCC cell line HepG2 were not reversed by the inhibition of FOXO3a transcriptional activity (Fig. 6C). These data suggest that the FOXO3a protein is a downstream mediator of AKT and ERK1/2 kinases.
Effects of FOXO3a transcription factor on the regulation of pro-apoptotic effects of VB1. AKT and ERK1/2 kinase have been shown to regulate the phosphorylation of FOXO3a protein $(24,25)$. We measured the phosphorylation of FOXO3a protein using western blot analysis. As shown in Fig. 6A, VB1 inhibited the phosphorylation of FOXO3a protein. However, VB1 had no effect on the expression of total FOXO3a protein (Fig. 7A).

Western blot analysis showed that FOXO3a was downregulated after transfection with specific siRNA targeting FOXO3a 
A

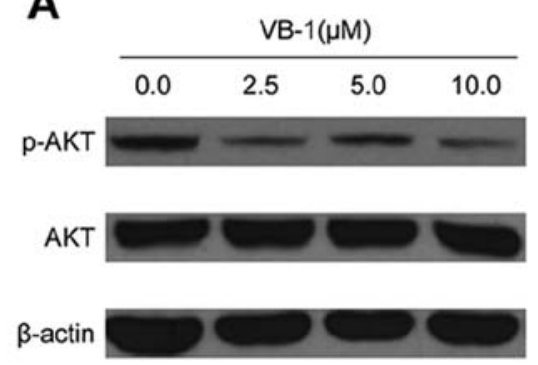

B

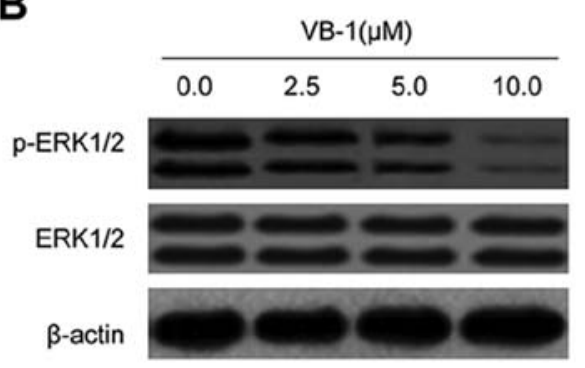

Figure 4. VB1 decreases the phosphorylation of AKT and ERK1/2 in HepG2 cells. (A) HepG2 cells were exposed to the indicated concentrations of VB1 for $24 \mathrm{~h}$, and western blotting of p-AKT, total-AKT and the loading control $\beta$-actin was undertaken. (B) HepG2 cells were exposed to the indicated concentrations of VB1 for $24 \mathrm{~h}$, and western blotting of p-ERK1/2, total-ERK1/2 and the loading control $\beta$-actin was undertaken.

A
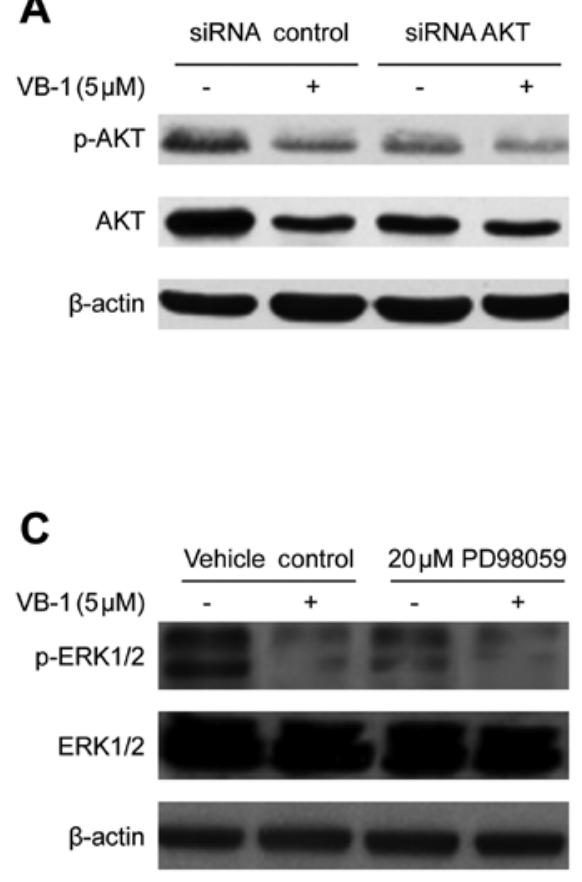

B

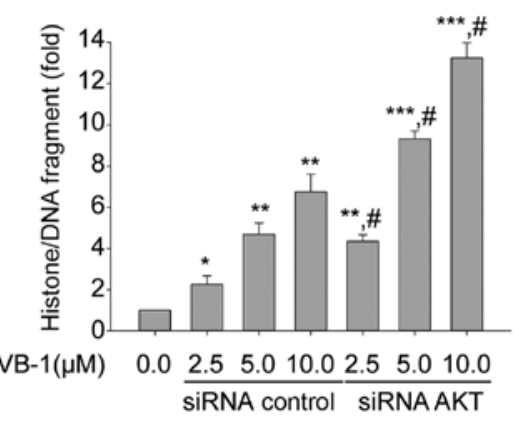

D

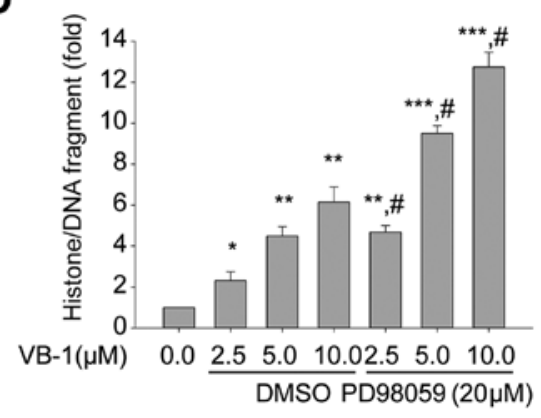

Figure 5. Effects of the knockdown of AKT1 and by siRNA and MEK1/2 inhibitor (PD98059) on apoptosis in HepG2 cells in the presence or absence of VB1. (A) HepG2 cells were transfected with $100 \mathrm{nM}$ of siRNA control or the siRNA duplexes against AKT mRNA. Twenty-four hours after the transfection, the cells were exposed to $5 \mu \mathrm{M}$ VB1 for $24 \mathrm{~h}$. Western blotting of p-AKT, AKT was performed to confirm the downregulation of AKT by siRNA transfection. $\beta$-actin was used as the loading control. (B) HepG2 cells were transfected with $100 \mathrm{nM}$ of siRNA control or the siRNA duplexes against AKT mRNA Twenty-four hours after the transfection, the cells were exposed to the indicated concentration of VB1 for $24 \mathrm{~h}$. Histone/DNA fragment of HepG2 cells were measured by the cell apoptosis ELISA detection kit. Data shown are means $\pm \mathrm{SD}$. $(\mathrm{n}=3) .{ }^{*} \mathrm{P}<0.05,{ }^{* *} \mathrm{P}<0.01,{ }^{* * * *} \mathrm{P}<0.001$ vs. $0.1 \%$ DMSO or $0 \mathrm{~h}$. ${ }^{\#} \mathrm{P}<0.05$ vs. the same concentration of VB1 in combination with siRNA control transfection. (C) HepG2 cells were exposed to $5 \mu \mathrm{M}$ VB1 for $24 \mathrm{~h}$ in the presence or absence of $20 \mu \mathrm{M}$ PD98059. Western blotting of p-ERK1/2, total-ERK1/2 and the loading control $\beta$-actin was undertaken. (D) HepG2 cells were exposed to the indicated concentrations of VB1 for $24 \mathrm{~h}$ in the presence or absence of $20 \mu \mathrm{M}$ PD98059. Histone/DNA fragment of HepG2 cells was measured using a cell apoptosis ELISA detection kit. Data shown are means $\pm \mathrm{SD}$. $(\mathrm{n}=3) .{ }^{*} \mathrm{P}<0.05,{ }^{* *} \mathrm{P}<0.01,{ }^{* * * *} \mathrm{P}<0.001$ vs. $0.1 \%$ DMSO or $0 \mathrm{~h} .{ }^{*} \mathrm{P}<0.05$ vs. the same concentration of VB1 alone. VB1, purified vitexin compound 1; siRNA, small interfering RNA.

in HepG2 cells (Fig. 7B). The results also indicated that inhibition of FOXO3a expression by siRNA inhibited VB1-induced apoptosis (Fig. 7C). These data suggest that VB1-induced apoptosis may be mediated by regulation of FOXO3a, and that inhibition of FOXO3a inhibits VB1-induced apoptosis.

Effects of VBI on the expression of FOXO3a downstream apoptosis-associated target genes. It has been reported that activation of $\mathrm{FOXO} 3 \mathrm{a}$ results in upregulation of the apop- tosis-associated target gene products Bim, TRAIL, DR4 and DR5 proteins (20). We, therefore, examined the effect of VB1 on the expression of Bim, TRAIL, DR4 and DR5 proteins. These genes are direct targets of FOXO3a transcription factor. The results indicated that VB1 induced the expression of Bim, TRAIL, DR4 and DR5 proteins (Fig. 8A-D). These data further suggest that VB1-induced apoptosis in HepG2 cells is mediated through mechanisms that involve the activation of FOXO3a transcription factor. 


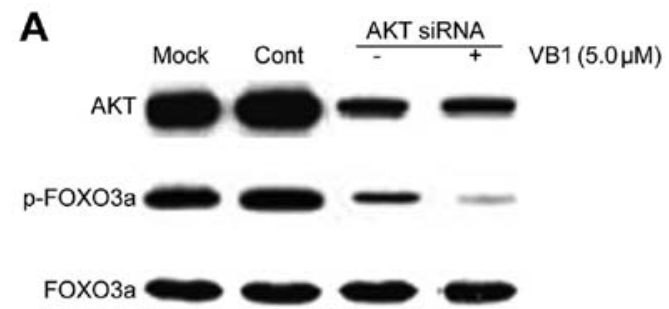

B
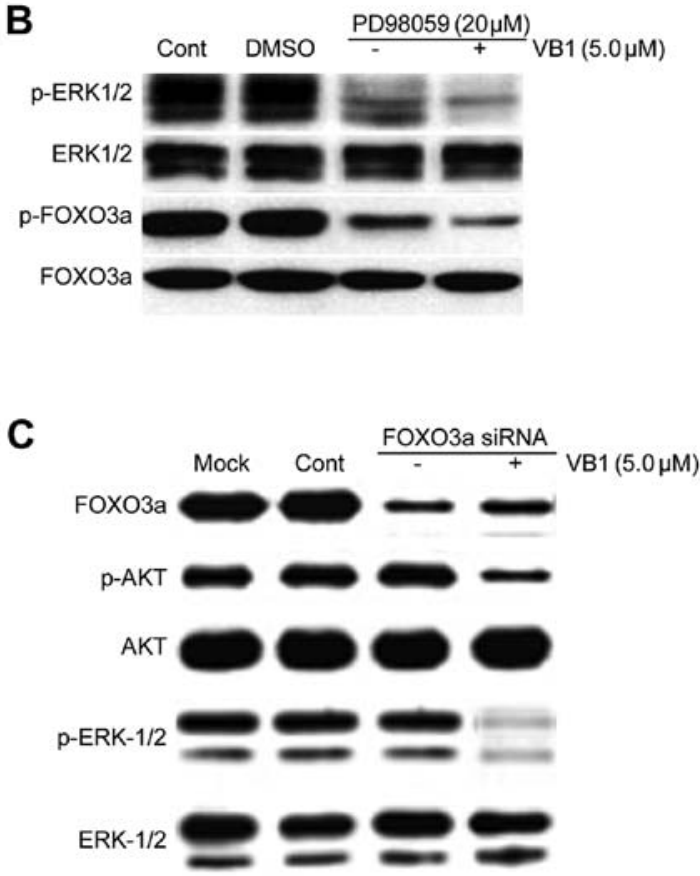

Figure 6. Effects of inhibition of AKT and ERK1/2 kinases on VB1-induced FOXO3a transcriptional activation. (A) HepG2 cells were transfected with $100 \mathrm{nM}$ of siRNA control or the siRNA duplexes against AKT mRNA Twenty-four hours after transfection, the cells were treated with $5 \mu \mathrm{M}$ VB1 for $24 \mathrm{~h}$. Western blotting of AKT, p-FOXO3a and FOXO3a was performed to confirm the effects of the downregulation of AKT by siRNA transfection. (B) HepG2 cells were pretreated with $20 \mu \mathrm{mol} / 1$ PD98059. Two hours after incubation, the cells were treated with $5 \mu \mathrm{M}$ VB1 for $24 \mathrm{~h}$. Western blotting of p-ERK1/2, ERK1/2, p-FOXO3a and FOXO3a was performed to confirm the effects of the inhibition of ERK1/2. (C) HepG2 cells were transfected with $100 \mathrm{nM}$ of siRNA control or the siRNA duplexes against FOXO3a mRNA. Twenty-four hours after transfection, the cells were treated with $5 \mu \mathrm{M}$ VB1 for $24 \mathrm{~h}$. Western blotting of FOXO3a, p-AKT, AKT, p-ERK1/2 and ERK1/2 was carried out to confirm the effects of the downregulation of FOXO3a by siRNA transfection. VB1, purified vitexin compound 1; siRNA, small interfering RNA.

\section{Discussion}

In the present study, we demonstrated that VB1-induced apoptosis in HCC cancer cells appears to be mediated through inhibition of AKT and ERK1/2 kinases and activation of FOXO3a transcription factor. Our results showed that inhibition of AKT and ERK1/2 kinases increased VB1-induced apoptosis, and that inhibition of FOXO3a transcription factor by siRNA blocked VB1-induced apoptosis. We also showed that VB1 upregulated Bim, TRAIL, DR4 and DR5. These findings indicate that VB1 induces apoptosis by inhibiting AKT and ERK1/2 kinases which results in activation of FOXO3a transcription factor in HCC cells. Apoptotic cells share a
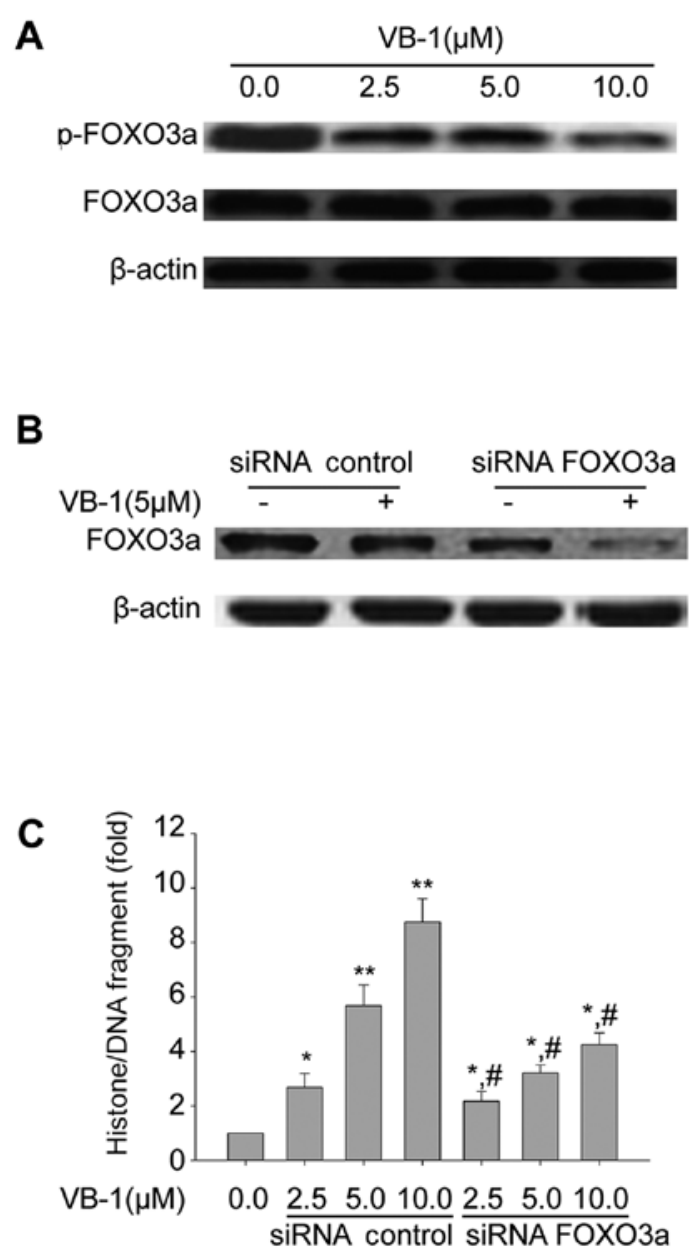

Figure 7. Effects of the knockdown of FOXO3a by siRNA on apoptosis in HepG2 cells in the presence or absence of VB1. (A) HepG2 cells were exposed to the indicated concentrations of VB1 for $24 \mathrm{~h}$, and western blotting of p-FOXO3a, total-FOXO3a and the loading control $\beta$-actin was undertaken. (B) HepG2 cells were transfected with $100 \mathrm{nM}$ of siRNA control or the siRNA duplexes against FOXO3a mRNA. Twenty-four hours after transfection, the cells were treated with $5 \mu \mathrm{M}$ VB1 for $24 \mathrm{~h}$. Western blotting of FOXO3a was performed to confirm the downregulation of FOXO3a by siRNA transfection. $\beta$-actin was used as the loading control. (C) HepG2 cells were transfected with $100 \mathrm{nM}$ of siRNA control or the siRNA duplexes against FOXO3a mRNA. Twenty-four hours after the transfection, the cells were exposed to the indicated concentration of VB1 for $24 \mathrm{~h}$. The histone/DNA fragment of HepG2 cells was measured using a cell apoptosis ELISA detection kit. Data shown are means $\pm \mathrm{SD}(\mathrm{n}=3)$. ${ }^{*} \mathrm{P}<0.05,{ }^{* *} \mathrm{P}<0.01$ vs. $0.1 \%$ DMSO or $0 \mathrm{~h} .{ }^{*} \mathrm{P}<0.05$ vs. the same concentration of VB1 in combination with siRNA control transfection. siRNA, small interfering RNA; VB1, purified vitexin compound 1 .

number of common characteristics. Caspase-3 activation, an enhanced sub-G1 cell population, histone/DNA fragmentation and DNA ladders are regarded as specific indicators of apoptosis. In the present study, we demonstrated activation of caspase-3, an increase in the sub-G1 population, histone/DNA fragments and the presence of a DNA ladder in VB1-treated HepG2 cells, thus demonstrating that VB1 induces HCC cell apoptosis. Overall, these properties of VB1 strongly suggest that it may be used as a cancer chemopreventive agent.

Caspase activation is known to play an important role in apoptosis triggered by various pro-apoptotic signals $(28,29)$. It is generally recognized that there are two major apoptotic pathways: one involves the transduction of death signals 


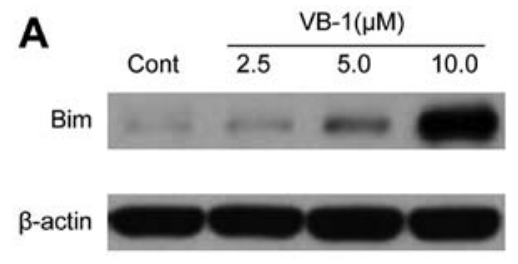

C

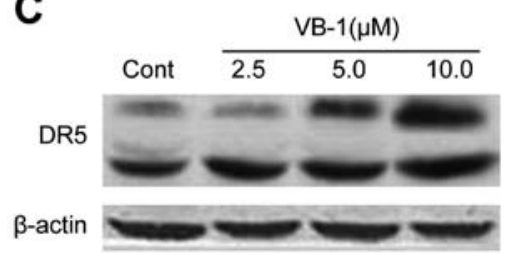

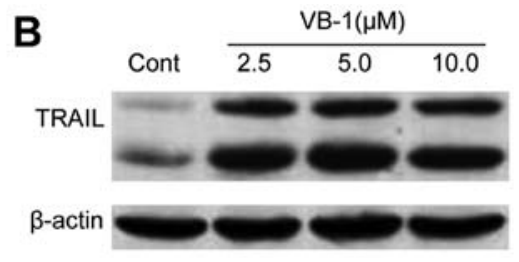

D

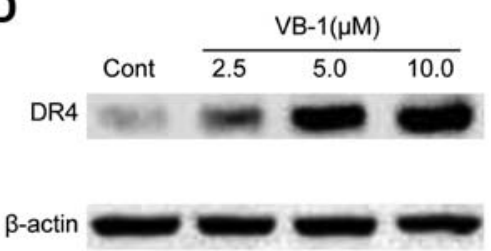

Figure 8. Effects of VB1 on the expression of Bim, TRAIL, DR4 and DR5 proteins in HepG2 cells. HepG2 cells were exposed to the indicated concentrations of VB1 for $24 \mathrm{~h}$, and western blotting of (A) Bim, (B) TRAIL, (C) DR5, (D) DR4 and the loading control $\beta$-actin was performed. VB1, purified vitexin compound 1.

through death receptors, and the other involves mitochondrial signaling $(28,30)$. Both pathways require ordered activation of a set of caspases, which cleave cellular substrates resulting in the morphological and biochemical changes that characterize apoptosis. Activation of caspase- 8 and -9 , have respectively been shown to play a central role in mediating apoptosis signaled by death receptors and by mitochondria. In the present study, we demonstrated that VB1 was able to activate caspase-3 -8 and -9 activity. The presence of the caspase inhibitor attenuated apoptosis induced by VB1, indicating that VB1-induced apoptosis was essentially dependent on the activation of caspase- $3,-8$ and -9 .

Forkhead O transcription factors (FOXO) factors have been shown to be dysregulated in a variety of tumor types, including HCC, owing to the constitutive activation of AKT and ERK1/2 kinases $(31,32)$. FOXOs are regulated by synthesis, phosphorylation, acetylation and ubiquitination at three different levels involving transcriptional activity, subcellular localization and stability (33). AKT and ERK kinases are thought to phosphorylate FOXO3a at different sites in response to growth factor and insulin stimulation. Phosphorylation of FOXO3a by these oncogenic kinases results in its translocation from the nucleus to cytoplasm where it is subsequently degraded $(34,35)$. Previous studies have shown that FOXO3a is at least one of the missing links connecting the two pathways. Inhibition of the PI3K/Akt/mTOR and MEK/ERK pathways has been shown to result in loss of phosphorylation at Akt and ERK phosphorylation sites, accompanied by nuclear accumulation and increased transcriptional activity of FOXO3a (35). In the present study, we demonstrated that inhibition of AKT and ERK1/2 kinases acts synergistically to regulate apoptotic effects of VB1 through activation of FOXO3a transcription factor. VB1 inhibited the accumulation of phosphorylated FOXO3a protein.

FOXO factors are also critical for the regulation of cell cycle arrest, cell death and DNA damage repair. Consequently, inactivation of FOXO proteins has been shown to be associated with tumorigenesis in breast and prostate cancer, glioblastoma, leukemia and hepatocellular carcinoma $(20,36)$. The FOXO subfamily contains 4 members (FOXO1, FOXO3, FOXO4 and FOXO6), which activate or repress multiple genes such as Bim, TRAIL, DR4, DR5 and survivin that are involved in apop- tosis (37-41). Our findings demonstrate that VB1 regulates the expression of FOXO3a apoptosis-associated target genes. We also showed that inhibition of FOXO3a by siRNA blocked the apoptotic effects of VB1. These results suggest that FOXO3a transcription factor mediated the anti-proliferative and pro-apoptotic effects of VB1.

Previous studies indicated that the effects of anticancer agents may be regulated through activation of FOXO transcription factors (41-43). In addition to their effects on cell cycle, apoptosis, differentiation and reactive oxygen species, FOXO proteins have also been implicated in the negative regulation of angiogenesis. For example, resveratrol and ECGG inhibit angiogenesis through activation of FOXO $(44,45)$. Collectively, these findings demonstrate that dephosphorylation and activation of FOXO by inhibition of AKT and ERK kinases may have significant implications for the treatment and prevention of, among others, diabetic complications, cardiovascular diseases and malignant neoplastic disease.

In summary, our results suggest that VB1 induces apoptosis through regulation of FOXO3a transcription factor in human HCC cells. Pharmacological and genetic inhibition of AKT and ERK1/2 kinases may act synergistically with VB1 to induce FOXO3a transcriptional activity which are mediated by dephosphorylation. Although a variety of clinical and in vivo studies are required to explore the therapeutic potential of VB1, our present study, together with our previous studies, indicates that VB1 may be a potential agent for further development in the prevention and treatment of HCC as well as other types of cancer.

\section{Acknowledgements}

The authors thank Dr Jian-Guo Cao (Medical College, Hunan Normal University, Changsha, Hunan, China) for the critical input into the manuscript. The present study was supported by Major State Science and Technology Special Purpose of China (2009ZX09102-109), the National Science and Technology Major Projects for 'Major New Drugs Innovation and Development' (2012ZX09303014001), the International Science and Technology Cooperation Program of China (2011DFA30620), and the Hunan Province Science and Technology Project (2009FG3142). 


\section{References}

1. Zhu AX: Molecularly targeted therapy for advanced hepatocellular carcinoma in 2012: current status and future perspectives. Semin Oncol 39: 493-502, 2012.

2. Siegel R, Naishadham D and Jemal A: Cancer statistics, 2013. CA Cancer J Clin 63: 11-30, 2013.

3. Padma S, Martinie JB and Iannitti DA: Liver tumor ablation: percutaneous and open approaches. J Surg Oncol 100: 619-634, 2009.

4. Ichida T, Van Thiel DH and Hassanein T: The medical management of hepatocellular carcinoma (HCC) in Japan: a review with implications for HCC seen in the west. Hepatogastroenterology 43 : $1575-1583,1996$.

5. Bruix J, Sherman M; Practice Guidelines Committee, American Association for the Study of Liver Diseases: Management of hepatocellular carcinoma. Hepatology 42: 1208-1236, 2005.

6. Llovet JM, Real MI, Montaña X, et al: Arterial embolisation or chemoembolisation versus symptomatic treatment in patients with unresectable hepatocellular carcinoma: a randomised controlled trial. Lancet 359: 1734-1739, 2002

7. Choo CY, Sulong NY, Man F and Wong TW: Vitexin and isovitexin from the leaves of Ficus deltoidea with in-vivo $\alpha$-glucosidase inhibition. J Ethnopharmacol 142: 776-781, 2012.

8. Knipping K, Garssen J and van't Land B: An evaluation of the inhibitory effects against rotavirus infection of edible plant extracts. Virol J 9: 137, 2012.

9. Sahreen S, Khan MR and Khan RA: Hepatoprotective effects of methanol extract of Carissa opaca leaves on $\mathrm{CCl}_{4}$-induced damage in rat. BMC Complement Altern Med 11: 48, 2011

10. Lee CY, Chien YS, Chiu TH, et al: Apoptosis triggered by vitexin in U937 human leukemia cells via a mitochondrial signaling pathway. Oncol Rep 28: 1883-1888, 2012.

11. Papi A, Farabegoli F, Iori R, et al: Vitexin-2-O-xyloside, raphasatin and (-)-epigallocatechin-3-gallate synergistically affect cel growth and apoptosis of colon cancer cells. Food Chem 138 : $1521-1530,2013$.

12. Xin H, Kong Y, Wang Y, et al: Lignans extracted from Vitex negundo possess cytotoxic activity by G2/M phase cell cycle arrest and apoptosis induction. Phytomedicine 20: 640-647, 2013

13. Zhou Y, Liu YE, Cao J, et al: Vitexins, nature-derived lignan compounds, induce apoptosis and suppress tumor growth. Clin Cancer Res 15: 5161-5169, 2009.

14. Tan Z, Zhang Y, Deng J, Zeng G and Zhang Y: Purified vitexin compound 1 suppresses tumor growth and induces cell apoptosis in a mouse model of human choriocarcinoma. Int J Gynecol Cancer 22: 360-366, 2012.

15. McCubrey JA, Steelman LS, Chappell WH, et al: Mutations and deregulation of Ras/Raf/MEK/ERK and PI3K/PTEN/Akt/ mTOR cascades which alter therapy response. Oncotarget 3: 954-987, 2012

16. Muntané J, De la Rosa AJ, Docobo F, Garcia-Carbonero R and Padillo FJ: Targeting tyrosine kinase receptors in hepatocellular carcinoma. Curr Cancer Drug Targets 13: 300-312, 2013.

17. Cervello M, McCubrey JA, Cusimano A, Lampiasi N, Azzolina A and Montalto G: Targeted therapy for hepatocellular carcinoma: novel agents on the horizon. Oncotarget 3: 236-260, 2012

18. Cai C, Teng L, Vu D, et al: The heme oxygenase 1 inducer (CoPP) protects human cardiac stem cells against apoptosis through activation of the extracellular signal-regulated kinase (ERK)/NRF2 signaling pathway and cytokine release. J Biol Chem 287 33720-33732, 2012

19. Xu X, Fan Z, Kang L, et al: Hepatitis B virus X protein represses miRNA-148a to enhance tumorigenesis. J Clin Invest 123 630-645, 2013

20. Yang JY and Hung MC: Deciphering the role of forkhead transcription factors in cancer therapy. Curr Drug Targets 12: 1284-1290, 2011

21. Arden KC: Multiple roles of FOXO transcription factors in mammalian cells point to multiple roles in cancer. Exp Gerontol 41: 709-717, 2006.

22. $\mathrm{Hu} \mathrm{MC}$, Lee DF, Xia W, et al: I $\mathrm{B}$ kinase promotes tumorigenesis through inhibition of forkhead FOXO3a. Cell 117: 225-237, 2004.

23. Potente M, Urbich C, Sasaki K, et al: Involvement of Foxo transcription factors in angiogenesis and postnatal neovascularization. J Clin Invest 115: 2382-2392, 2005.

24. Brunet A, Bonni A, Zigmond MJ, et al: Akt promotes cell survival by phosphorylating and inhibiting a Forkhead transcription factor. Cell 96: 857-868, 1999.
25. Yang JY,Zong CS, Xia W, et al: ERK promotes tumorigenesis by inhibiting FOXO3a via MDM2-mediated degradation. Nat Cell Biol 10: 138-148, 2008.

26. Kim SH, Jeong JH, Kim TI, Kim SW and Bull DA: VEGF siRNA delivery system using arginine-grafted bioreducible poly(disulfide amine). Mol Pharm 6: 718-726, 2009.

27. Du J, Gao S, Luo J, et al: Effective inhibition of foot-and-mouth disease virus (FMDV) replication in vitro by vector-delivered microRNAs targeting the 3D gene. Virol J 8: 292, 2011

28. Guerrero AD, Schmitz I, Chen M and Wang J: Promotion of caspase activation by caspase-9-mediated feedback amplification of mitochondrial damage. J Clin Cell Immunol 3: pii: 1000126 , 2012.

29. Ranjan K, Surolia A and Pathak C: Apoptotic potential of Fas-associated death domain on regulation of cell death regulatory protein cFLIP and death receptor mediated apoptosis in HEK 293T cells. J Cell Commun Signal 6: 155-168, 2012.

30. Yamasaki $Y$, Yamasaki $M$, Tachibana $H$ and Yamada $K$ : Important role of $\beta 1$-integrin in fucoidan-induced apoptosis via caspase-8 activation. Biosci Biotechnol Biochem 76: 1163-1168, 2012.

31. Kornblau SM, Singh N, Qiu Y, Chen W, Zhang N and Coombes KR: Highly phosphorylated FOXO3A is an adverse prognostic factor in acute myeloid leukemia. Clin Cancer Res 16 $1865-1874,2010$

32. Vadlakonda L, Pasupuleti M and Pallu R: Role of PI3K-AKTmTOR and Wnt signaling pathways in transition of G1-S phase of cell cycle in cancer cells. Front Oncol 3: 85, 2013.

33. Pardo PS, Lopez MA and Boriek AM: FOXO transcription factors are mechanosensitive and their regulation is altered with aging in the respiratory pump. Am J Physiol Cell Physiol 294: C1056-C1066, 2008.

34. Nho RS, Hergert P, Kahm J, Jessurun J and Henke C: Pathological alteration of FoxO3a activity promotes idiopathic pulmonary fibrosis fibroblast proliferation on type I collagen matrix. Am J Pathol 179: 2420-2430, 2011.

35. Wang X, Chen WR and Xing D: A pathway from JNK through decreased ERK and Akt activities for FOXO3a nuclear translocation in response to UV irradiation. J Cell Physiol 227: 1168-1178, 2012.

36. Xie C, Song LB, Wu JH, et al: Upregulator of cell proliferation predicts poor prognosis in hepatocellular carcinoma and contributes to hepatocarcinogenesis by downregulating FOXO3a. PLoS One 7: e40607, 2012

37. Ghosh AP, Klocke BJ, Ballestas ME and Roth KA: CHOP potentially co-operates with $\mathrm{FOXO} 3 \mathrm{a}$ in neuronal cells to regulate PUMA and BIM expression in response to ER stress. PLoS One 7: e39586, 2012

38. Zhao XC, Cao XC, Liu F, Quan MF, Ren KQ and Cao JG Regulation of the FOXO3a/Bim signaling pathway by 5,7-dihydroxy-8-nitrochrysin in MDA-MB-453 breast cancer cells. Oncol Lett 5: 929-934, 2013.

39. Cunha DA, Igoillo-Esteve M, Gurzov EN, et al: Death protein 5 and p53-upregulated modulator of apoptosis mediate the endoplasmic reticulum stress-mitochondrial dialog triggering lipotoxic rodent and human $\beta$-cell apoptosis. Diabetes 61: 2763-2775, 2012

40. He L, Yang X, Cao X, Liu F, Quan M and Cao J: Casticin induces growth suppression and cell cycle arrest through activation of FOXO3a in hepatocellular carcinoma. Oncol Rep 29: 103-108, 2013.

41. Chen Q, Ganapathy S, Singh KP, Shankar S and Srivastava RK: Resveratrol induces growth arrest and apoptosis through activation of FOXO transcription factors in prostate cancer cells. PLoS One 5: e15288, 2010

42. Boreddy SR, Pramanik KC and Srivastava SK: Pancreatic tumor suppression by benzyl isothiocyanate is associated with inhibition of PI3K/AKT/FOXO pathway. Clin Cancer Res 17: 1784-1795, 2011.

43. Roy SK, Srivastava RK and Shankar S: Inhibition of PI3K/AKT and MAPK/ERK pathways causes activation of FOXO transcription factor, leading to cell cycle arrest and apoptosis in pancreatic cancer. J Mol Signal 5: 10, 2010.

44. Shankar S, Marsh L and Srivastava RK: EGCG inhibits growth of human pancreatic tumors orthotopically implanted in Balb C nude mice through modulation of FKHRL1/FOXO3a and neuropilin. Mol Cell Biochem 372: 83-94, 2013.

45. Srivastava RK, Unterman TG and Shankar S: FOXO transcription factors and VEGF neutralizing antibody enhance antiangiogenic effects of resveratrol. Mol Cell Biochem 337: 201-212, 2010. 